\title{
As tecnologias da informação e comunicação no ensino de Biologia: Aproximações
}

\section{teóricas}

\author{
Information and communication technologies in Biology education: Theoretical approaches \\ Tecnologías de la información y la comunicación en la educación en Biología: Enfoques teóricos
}

Recebido: 12/03/2021 | Revisado: 18/03/2021 | Aceito: 20/03/2021 | Publicado: 27/03/2021

Ítalo Câmara de Almeida
ORCID: https://orcid.org/0000-0002-3349-1796
Universidade Federal do Espírito Santo, Brasil
E-mail: almeidaicvet@gmail.com
Luciana Canário Mendes
ORCID: https://orcid.org/0000-0003-1239-3692
Instituto Federal de Educação, Ciência e Tecnologia do Norte de Minas Gerais, Brasil
E-mail: lucianacanario@gmail.com
Ricael Spirandeli Rocha
ORCID: https://orcid.org/0000-0003-3190-7513
Instituto Federal de Educação, Ciência e Tecnologia de Minas Gerais, Brasil
E-mail: ricael.edu @gmail.com

\begin{abstract}
Resumo
Dada a importância da integração das Tecnologias da Informação e Comunicação (TIC) em um contexto de ensino de Biologia, o presente estudo teve como objetivo caracterizar as principais tecnologias digitais e tendências de referenciais teóricos (fundamentação teórica e revisão de literatura) de estudos que investigam o uso de tecnologias na educação, enfatizando o ensino de ciências e biologia. Nele, apresentamos uma reflexão de um conjunto de estudos, durante os quais as tecnologias digitais assumiram uma presença crescente dentro da sala de aula na educação básica. A revisão bibliográfica foi constituída principalmente de livros, artigos científicos, teses e dissertações, sobretudo de exemplares disponíveis online em plataformas de pesquisas como Scielo, CAPES e Google Acadêmico. Os principais descritores utilizados foram: tecnologias na educação, ensino de ciências e biologia, educação científica, TIC, educação básica. A pesquisa evidenciou que é imprescindível discutir as abordagens necessárias para capacitar o processo de indução e inovação constante na educação e aprendizagem. Alcançar o objetivo de integração significativa da tecnologia na sala de aula, não depende apenas de fatores relacionados à tecnologia, as crenças pedagógicas pessoais dos professores desempenham um papel fundamental em suas decisões pedagógicas sobre como integrar a tecnologia em suas práticas de sala de aula. O grande desafio está em encontrar formas positivas e viáveis de integrar as TIC no processo de ensino-aprendizagem, no quadro dos currículos atuais, da situação profissional dos professores e das condições concretas de atuação em cada escola.
\end{abstract}

Palavras-chave: Tecnologias de informação e comunicação; Ensino e aprendizagem; Biologia.

\begin{abstract}
Given the importance of integrating Information and Communication Technologies (ICT) in a Biology teaching context, the present study aimed to characterize the main digital technologies and trends in theoretical frameworks (theoretical foundation and literature review) of studies that investigate the use of technologies in education, emphasizing the teaching of science and biology. In it, we present a reflection of a set of studies, during which digital technologies have assumed a growing presence within the classroom in basic education. The bibliographic review consisted mainly of books, scientific articles, theses and dissertations, especially copies available online on research platforms such as Scielo, CAPES and Google Scholar. The main descriptors used were: technologies in education, science and biology teaching, science education, ICT, basic education. The research showed that it is essential to discuss the approaches needed to enable the process of induction and constant innovation in education and learning. Achieving the goal of meaningful integration of technology in the classroom, does not depend only on factors related to technology, the personal pedagogical beliefs of teachers play a fundamental role in their pedagogical decisions on how to integrate technology in their classroom practices. The great challenge is to find positive and viable ways of integrating ICTs in the teaching-learning process, within the framework of the current curricula, the professional situation of teachers and the concrete conditions of performance in each school.
\end{abstract}

Keywords: Information and communication technologies; Teaching and learning; Biology. 


\begin{abstract}
Resumen
Dada la importancia de la integración de las Tecnologías de la Información y la Comunicación (TIC) en un contexto de enseñanza de la Biología, el presente estudio tuvo como objetivo caracterizar las principales tecnologías digitales y tendencias en los marcos teóricos (fundamentación teórica y revisión de la literatura) de estudios que investigan el uso de las tecnologías en la educación, haciendo énfasis en la enseñanza de la ciencia. y biología. En él, presentamos una reflexión de un conjunto de estudios, durante los cuales las tecnologías digitales han asumido una presencia creciente dentro del aula en la educación básica. La revisión bibliográfica estuvo compuesta principalmente por libros, artículos científicos, tesis y disertaciones, especialmente copias disponibles en línea en plataformas de investigación como Scielo, CAPES y Google Scholar. Los principales descriptores utilizados fueron: tecnologías en la educación, enseñanza de la ciencia y la biología, educación científica, TIC, educación básica. La investigación mostró que es fundamental discutir los enfoques necesarios para posibilitar el proceso de inducción y la innovación constante en la educación y el aprendizaje. Alcanzar el objetivo de la integración significativa de la tecnología en el aula, no depende solo de factores relacionados con la tecnología, las creencias pedagógicas personales de los docentes juegan un papel fundamental en sus decisiones pedagógicas sobre cómo integrar la tecnología en sus prácticas de aula. El gran desafío es encontrar formas positivas y viables de integrar las TIC en el proceso de enseñanza-aprendizaje, en el marco de los planes de estudio actuales, la situación profesional de los docentes y las condiciones concretas de desempeño en cada escuela.
\end{abstract}

Palabras clave: Tecnologías de la información y la comunicación; Enseñando y aprendiendo; Biología.

\title{
1. Introdução
}

O mundo atual é marcado por uma série de mudanças que estão afetando o modo de ver, viver, ser e de pensar do homem. Esse movimento, cujo lado mais explorado e comentado é denominado globalização, vem-se acirrando e se acelerando nas últimas décadas. Esse processo tem ocorrido em paralelo ao avanço tecnológico, mais intrinsecamente associado à evolução das tecnologias da informação e da comunicação (TIC).

A sociedade do século XX e início do século XXI vivem momentos de grandes transformações, o tempo já não tem o mesmo significado, as distâncias já não representam mais limitações significativas para a comunicação e intercâmbio entre as pessoas e a sociedade.

Neste contexto, a educação é fundamental, pois deve proporcionar ao ser humano o seu desenvolvimento integral, tanto no que se refere aos conteúdos curriculares historicamente acumulados, bem como sua formação para o convívio em sociedade e atendimento a essas demandas.

Ao passo que a técnica se constitui como um conjunto de regras compendiadas externamente que indicam como determinada atividade deve ser realizada, ela implica repetitividade. E quando uma técnica deriva do conhecimento científico, ou seja, quando ela se fundamenta em princípios cientificamente estabelecidos que se aplicam ao planejamento, a construção e a utilização de um equipamento em um determinado tipo de atividade, denomina-se tecnologia (Saviani, 2011).

Complementarmente Kenski (2012) destaca que conceito de tecnologias engloba a totalidade de coisas que a engenhosidade do cérebro humano conseguiu criar em todas as épocas, suas formas de uso e suas aplicações. Há que se destacar que muitas tecnologias ao nosso redor não são máquinas, como por exemplo, as próteses e os medicamentos.

É preciso recuperar McLuhan (1996), que afirma que as tecnologias se tornam poucos visíveis quanto mais elas se tornam familiares. À medida que incorporamos o uso de tecnologias na vida cotidiana já não nos preocupamos tanto com o seu uso. Elas se tornam “invisíveis”, já não nos causam estranhamento.

Essas mudanças refletem, por sua vez, na organização e na natureza do trabalho, e na produção e no consumo de bens. Assim, também a educação e o "sistema" educacional têm sido pressionados para caminhar por novos rumos. Para Kenski (2012) este é o duplo desafio da educação: adaptar-se aos avanços das tecnologias e orientar o caminho dos educandos para o domínio e a apropriação crítica destes novos mecanismos.

$\mathrm{Na}$ tentativa de adaptação a essas demandas tecnológicas, os professores têm tentando promover a aprendizagem mais centrada no aluno, estimula a interação dos alunos com o professor e facilita a aprendizagem cooperativa (Sivin et al., 2000). 
Dentre os diversos campos científicos que buscam integrar as TIC aos processos de ensino-aprendizagem, destaca-se a Biologia, por fazer parte das nossas atividades cotidianas, bem como pelo entendimento de que é uma ciência que permite que os alunos tenham consciência da existência e da influência da natureza em seu cotidiano e se vejam com poderes para se responsabilizar pela atenção e conservação do meio ambiente (Yassanne, 2014).

Em vista disso, a presente pesquisa teve como objetivo analisar quais os recursos tecnológicos podem ser atrelados aos métodos de ensino que podem proporcionar uma aprendizagem significativa no ensino de Biologia na Educação Básica.

\section{Metodologia}

Como procedimento metodológico foi utilizada a revisão bibliográfica (constituída principalmente de livros, artigos científicos, teses e dissertações), sobretudo de exemplares disponíveis online em plataformas de pesquisas como Scielo, CAPES e Google Acadêmico. Tendo como foco a pesquisa de natureza qualitativa, caracterizada pela importante interpretação do pesquisador sobre o fenômeno em estudo (Pereira et al., 2018). Os principais descritores utilizados foram: <tecnologias na educação>, 〈ensino de ciências e biologia〉, 〈educação científica〉, 〈TIC〉, <educação básica〉.

\section{Resultados e Discussão}

As tecnologias digitais se tornaram parte integrante da vida humana, abrindo caminho para muitas inovações. Essas inovações e desenvolvimentos em tecnologia, por sua vez, também levaram à introdução de ideias sobre o seu uso em diferentes áreas, como a educação. Para o século XXI, as habilidades exigem o domínio das áreas de conhecimento com tecnologia para melhorar as habilidades de aprendizado e raciocínio (Nicolle \& Lou, 2008).

$\mathrm{O}$ rápido aumento na disponibilidade e acessibilidade de tecnologias interativas contribuiu para promover a aprendizagem em ciências e biologia, tanto na educação básica como no ensino superior. Os alunos estão ansiosos para experimentar diferentes metodologias para apoiar seu aprendizado, em grande parte porque são hábeis no uso de tecnologia e gostam de usar aplicativos e jogos. Tais métodos na educação aumentam a motivação e o envolvimento do aluno (Barrio et al., 2016; Wang \& Lieberoth, 2016), especialmente em circunstâncias em que o estilo de aula convencional, centrada no professor, é ressentido pelos alunos ao induzirem o tédio (Graham, 2015; Cheong et al., 2013; Roehl et al., 2013).

A proposta feita pela Organização das Nações Unidas para Educação, Ciência e Cultura (UNESCO) para melhorar as estratégias de ensino de Biologia incentivou a diversificação do conteúdo das aulas, métodos e compartilhamento de conhecimento com a tecnologia nos cursos de biologia (Anderson et al., 2002). Nesse sentido, essas diversificações podem ser utilizadas como métodos de ensino que incorporam a tecnologia nos cursos de biologia. Assim, os alunos terão que aprimorar suas habilidades de raciocínio e aprendizagem, bem como sua alfabetização em tecnologias, sobretudo em tecnologias de informação e comunicação, de forma a cumprir as propostas dos métodos de ensino.

Quanto aos efeitos da tecnologia sobre os alunos, ela tem vantagens e desvantagens em termos de conhecimento e atitudes (Incantalupo et al., 2014). Uma das vantagens de usar a tecnologia é melhorar as habilidades de pensamento de nível superior dos alunos, além de suas habilidades de investigação (Costley, 2014).

Embora os resultados da maioria dos estudos mostrem um impacto positivo da tecnologia no conhecimento e na atitude dos alunos, quando se trata de biologia, as diferenças de gênero criam uma desigualdade importante. Como resultado, os pesquisadores descobriram que há uma diferença significativa entre estudantes do sexo masculino e feminino em termos de suas atitudes em relação à essa nova metodologia. As alunas tendem a usar a tecnologia por meio da influência social, em vez de tomar suas próprias decisões. No entanto, os alunos são mais inclinados a tomar suas próprias decisões ao usar a tecnologia (Mazman et al., 2009). 
De acordo com o estudo de Incantalupo et al. (2014), percepção, interesse e diferenças de gênero podem afetar o comportamento e as atitudes dos alunos em relação à tecnologia. Há uma pequena diferença de percepção em relação ao gênero; enquanto as alunas consideram o computador uma ferramenta tecnológica, os homens o consideram um jogo. Mammes (2004) indica que as mulheres têm dificuldade em se conectar com a tecnologia, portanto, elas são mais relutantes em usar tecnologia ou ingressar em atividades tecnológicas.

As tecnologias digitais e TIC, que devem ser utilizadas pelos alunos, incluem ferramentas tecnológicas que são utilizadas do passado ao presente, como retroprojetor, rádio, televisão, computadores e internet. Essas são ferramentas poderosas para mudanças e reformas educacionais em ambientes de ensino e aprendizagem. Apesar da evidência atual indicar que, embora o uso da tecnologia durante o processo de ensino e aprendizagem esteja aumentando constantemente (Berrett et al., 2012; Inan \& Lowther, 2010), alcançar a integralidade no uso da tecnologia ainda é um processo complexo de mudança educacional. Isso é evidente pois o uso da tecnologia nas escolas ainda é extremamente variado e, em muitos casos, limitado (Spector, 2015).

De acordo com Olaore (2014), se o professor não adaptar os seus métodos de ensino para fazer o melhor uso das TIC, então o propósito de usá-las não se justifica. Também a atitude do estabelecimento educacional parece ter um efeito maior quando as pessoas que os dirigem não têm o conhecimento e experiência no uso das TIC.

Neste contexto, pesquisadores argumentaram que as práticas de sala de aula dos professores são altamente influenciadas por suas crenças pedagógicas. Desta forma, os professores selecionam e aplicam as tecnologias que se alinham com seus métodos e suas estratégias de ensino, e que também se alinham com as suas crenças existentes sobre a boa educação (Ertmer et al., 2015; Fives \& Gill, 2014). Dispositivos tecnológicos como computadores, tablets ou lousas interativas não ditam a abordagem pedagógica de uma pessoa (Lawless \& Pellegrino, 2007), em vez disso, cada dispositivo permite a implementação de uma variedade de abordagens de ensino e aprendizagem (Tondeur et al., 2008). Em outras palavras, o papel que a tecnologia desempenha nas salas de aula dos professores se relacionam com suas concepções da natureza do ensino e da aprendizagem.

\subsection{Tecnologia Educacional}

Entre os profissionais que se dedicam à educação, o termo "tecnologia educacional" é amplamente utilizado. Como resultado, pensa-se que o termo não deve ser introduzido em um padrão de definição. Pois, com o passar do tempo, observa-se que muitas das definições que foram definidas anteriormente, foram refutadas pelo aumento de informações e inovações. Em linhas gerais, a tecnologia educacional é a utilização bem-sucedida de tecnologia, aparelhos, sistemas ativos e procedimentos para ajudar na aprendizagem, execução e orientação do processo ensino-aprendizagem (Huang et al., 2019).

Segundo Spector (2015), pode ser considerada como definições e recursos da tecnologia educacional a implementação disciplinada de informações com o objetivo de melhorar a aprendizagem, instrução ou o desempenho dos alunos. Para Huang et al. (2019), a tecnologia educacional refere-se à utilização de dispositivos, avanços, formulários e procedimentos para melhorar os encontros de aprendizagem em uma variedade de ambientes como aprendizagem formal ou aprendizagem causal. Desta forma, a tecnologia educacional gira em torno dos recursos especializados e acadêmicos disponíveis para apoiar e orientar os alunos para melhorar o aprendizado.

A compreensão teórica, bem como a prática da tecnologia educacional, requer a construção e o refinamento contínuo do conhecimento por meio da pesquisa e da prática reflexiva. Segundo Bozkaya et al. (2012), os estudos sobre tecnologia educacional costumavam se concentrar em instrumentos educacionais até os anos 60 , desde então a pesquisa educacional passou a focar mais no processo, estudando os efeitos do uso da tecnologia na aprendizagem e no ensino. 
Contudo, há um contínuo desenvolvimento e alterações na tecnologia e, portanto, na educação. Desta forma, os estudos sobre tecnologias educacionais evoluem nesse sentido, e implementam o esclarecimento dos conteúdos a serem aplicados na tecnologia educacional que ocupam um lugar muito amplo na educação. Portanto, o apoio a recursos, desempenho e ensino no uso de tecnologias educacionais deve ser facilitado e aprimorado (Yalim, 2021).

\subsection{Ferramentas em tecnologia educacional}

Quando olhamos para a evolução tecnológica, vemos que a tecnologia tem um lugar no campo da educação. Muitos produtos tecnológicos que têm sido utilizados com o propósito de comunicação ainda são utilizados como ferramentas de tecnologia educacional. Os exemplos de ferramentas tecnológicas que têm um efeito notável na educação incluem; televisão, filmes instrucionais, microcomputadores, simulações interativas, internet, sistemas de ensino à distância, dentre outros (Ward et al., 2009).

Como o ambiente educacional está mudando rapidamente com essas tecnologias, é inevitável que os equipamentos educacionais sejam renovados junto com essas inovações, e assim a tecnologia será capaz de atender às necessidades de hoje. Huang et al. (2019), afirmam que as abordagens de tecnologia educacional evoluíram dos primeiros usos de recursos audiovisuais para computadores individuais e em rede, e agora evoluíram para incluir várias tecnologias móveis e inteligentes, bem como realidades virtuais e aumentadas, ambientes imersivos baseados em avatar, computação em nuvem e dispositivos com reconhecimento de localização.

Dentre os desenvolvimentos tecnológicos evoluídos, as redes sociais têm um lugar significativo na educação. Essas são ferramentas que melhoram a aprendizagem e, portanto, aumentam a satisfação dos alunos com a aula. Elas também reduzem a ansiedade dos alunos, melhoram suas habilidades de escrita e leitura e ajudam a integrar essas habilidades nos cursos de ciências e biologia (Ajjan \& Hartshorne, 2008).

Além disso, Arroyo (2011) afirma que as redes sociais apoiam a aprendizagem informal e são ferramentas de comunicação de alto desempenho em termos de estruturação da informação através da colaboração, relações sociais e compartilhamento de tarefas.

Da mesma forma, como resultado da evolução do computador e da internet, o uso da rede social, que é uma ferramenta de tecnologia educacional, também avançou. Ela assumiu um lugar tão importante quanto a mídia social, e pôde ser examinada em uma ampla variedade de ferramentas de tecnologia educacional. A mídia social, de fato, pode ser associada à educação, e também a jovens, a maioria dos quais são alunos da geração Y que continuam seu ensino superior (Bolton et al., 2013). Assim, os atuais desenvolvimentos tecnológicos têm efeito considerável na educação no século XXI.

A próxima fase da tecnologia educacional, em relação aos resultados positivos da combinação de mídia social e educação, é uma nova era em que as máquinas podem fazer perguntas, elas podem entrar em contato umas com as outras e buscar respostas para essas perguntas. Existem vários estudos sobre se essa experiência ainda não foi totalmente implementada ou qual será a situação da adoção mesmo se ela for implementada. Finalmente, talvez a atual tecnologia de inteligência artificial e a tecnologia de realidade virtual possam ser combinadas para dar uma nova direção à educação (Ochoa, 2016).

\subsection{Vantagens da tecnologia educacional}

Hoje é indiscutível que as TIC são amplamente utilizadas, aumentando o acúmulo de conhecimento, e por isso é necessário fazer mais uso desse conhecimento. Graças à tecnologia, as pessoas se comunicam muito rapidamente, fazem reuniões e palestras, compartilham recursos com outras pessoas e chegam a todas as informações que desejam. Nesta modernização, a mudança de qualificações esperadas dos indivíduos provoca ajustes na compreensão educacional, na importância da tecnologia e mesmo nos processos de ensino-aprendizagem das tecnologias educacionais. 
Com esses avanços, várias aplicações de tecnologia educacional podem ser usadas para eliminar algumas das deficiências encontradas na educação tradicional. Por exemplo, a utilização de tecnologia pode auxiliar na melhoria do aprendizado, pois diminuirá a quantidade de instruções diretas dadas aos alunos. Assim, o uso da tecnologia oferece aos educadores a chance de auxiliar os alunos a compreender as instruções mais facilmente e continuar com orientações adicionais para melhorar o aprendizado (Bingimlas, 2009).

As teorias e tecnologias de aprendizagem estão conectadas e entrelaçadas pelo processamento de informações. Como as tecnologias educacionais e as teorias de aprendizagem estão vinculadas, resultados positivos são obtidos na educação quando usadas em conjunto. Esses resultados positivos revelam como a tecnologia educacional pode ser transformada em uma vantagem. Algumas vantagens do uso das TIC na educação, são descritas por Yalim (2021), Santos et al. (2019), Bamiah et al. (2018), Costley (2014), Specto et al. (2014):

Melhorar o interesse dos alunos nos cursos;

Maior compreensão e motivação dos alunos;

Obter parecer objetivo para avaliar o ensino dos professores, processos, métodos e estrutura de cursos;

Permite ajudar os alunos a se sentirem privilegiados porque podem aprender sozinhos, tornando-os mais confiantes;

Oferece aos alunos a chance de se comunicarem pela internet e obterem as informações mais facilmente;

Ajuda a eliminar o problema de carregar livros, tornando as informações facilmente acessíveis por meio do uso de tecnologia educacional e suas ferramentas; dentre outras.

São muitas as vantagens do uso das tecnologias na educação, e é fato que a tecnologia pode melhorar o ensino e a aprendizagem em sala de aula. No entanto, a pesquisa realizada por Cunha \& Bizelli (2015) revelou que, também existem alguns obstáculos em relação ao uso de tais tecnologias. Tais estudos relatam que $88 \%$ dos professores entrevistados acreditam que o uso das tecnologias da informação e comunicação são importantes no contexto escolar para alunos do ensino médio, no entanto, $79 \%$ deles não se consideram preparados para utilizarem as TIC na sala de aula. Desta forma fica evidente que não é necessário investir apenas na tecnologia, mas também na capacitação dos professores para utilizarem as mesmas.

\subsection{Uso da tecnologia nas aulas de Biologia}

Nos últimos anos, o currículo de biologia mudou em relação as novas descobertas na ciência. Essas alterações também mostraram um aumento no uso das TIC no ensino e aprendizagem de ciências e biologia, com a mudança de perspectivas sobre a natureza da ciência e o papel da educação em ciências (Acosta et al., 2021).

Instruir sobre ciências, ao invés de experimentá-las, exigirá uma mudança notável em seus métodos educacionais, além do conhecimento e compreensão aprimorados por parte dos educadores. O uso de TIC em salas de aula de ciências foi pesquisado em muitos estudos (Gross et al., 2015; Waldrop et al., 2015; Mayes, 2011; Thompson et al., 2010), e estes relataram que existem benefícios potenciais, tais como comunicação e colaboração de acordo com os recursos pedagógicos utilizados. Os benefícios de usar as TIC como ferramentas para promover o ensino e a aprendizagem de ciências nas escolas incluem tornar a aprendizagem mais eficaz, fornecer parecer valioso, aumentar a confiança do professor e a eficácia pedagógica (Oliveira et al., 2019).

Embora se pensasse que as TIC não poderiam substituir as técnicas tradicionais de ensino em sala de aula, elas podem reforçar positivamente a compreensão dos conceitos e princípios do currículo de ciências (Incantalupo et al., 2014; Ross et al., 2010). No currículo de biologia, a utilização de ferramentas online e o enriquecimento do curso pelo professor é uma grande 
vantagem para alunos com diferentes estilos de aprendizagem. Portanto, a fim de alcançar a diferenciação e afastar-se dos métodos tradicionais de ensino, os educadores devem integrar a tecnologia ao currículo de biologia.

A biologia é um curso baseado em rotina, a rotina pode ser um problema para os alunos aprenderem ou se lembrarem do conteúdo da disciplina, uma vez que essa rotina pode deixar os alunos entediados. Muitas informações são passadas em uma sala de aula centrada no professor (Aydin \& Yel, 2011), e os alunos devem transferir todas essas informações para sua memória de curto prazo. Uma vez que os alunos não podem transferir o conhecimento de sua memória de curto prazo para a memória de longo prazo automaticamente, seu sucesso diminui. Portanto, a diferenciação nas técnicas de ensino e aprendizagem com o uso das tecnologias, deve ser empregada de forma a garantir que os alunos detenham as informações que aprenderam em curto prazo e que posteriormente vão ser transferidas para a memória de longo prazo (Kelley \& Whatson, 2013).

Para tanto, é importante usar as TIC como materiais audiovisuais dentro das atividades que os professores usam para ensinar o conteúdo do currículo de biologia, e desta forma apoiar os alunos com a tecnologia. Para alcançar a aprendizagem em salas de aula de biologia, professores e alunos devem ser capazes de usar essas ferramentas de forma eficiente (Akram \& Malik, 2012). Além disso, os alunos devem trabalhar de forma colaborativa para apoiar a aprendizagem uns dos outros à medida que usam essas ferramentas, o que tornará a educação mais centrada no aluno e ajudará a transferir novos conhecimentos para a memória de longo prazo (Hannafin \& Hill, 2002).

\subsection{A utilização responsável das TIC: possibilidades e desafios}

O principal desafio imposto pela interferência digital na educação, afirma Saviani (2011), reside na compreensão do significado histórico-social dos processos de informatização. Mais do que familiarizar os alunos com os procedimentos de digitação, o papel da educação escolar é permitir-lhes a compreensão dos princípios científicos que fundamentam esses procedimentos.

Essa nova demanda pode ser considerada um desafio, especialmente para o professor, porque a realidade escolar comporta um quadro em que as TIC não são compreendidas ou aceitas por eles, sequer como ferramentas pedagógicas potenciais na prática educacional com alunos. Mesmo presentes no cotidiano escolar, alguns professores ainda desconhecem suas potencialidades educativas. Aliadas aos problemas técnicos e operacionais, os professores desconhecem as potencialidades por inúmeros motivos, dentre eles, falta de tempo para realizar formação continuada dentro da jornada de trabalho, e formação inicial precária.

Segundo Belloni (2002), falta motivação dos professores para a realização de formação continuada, tendo em vista a ausência de incentivos de formação no plano de carreira e o nível de salários dessa categoria. A autora ainda destaca que, mesmo quando são oferecidos treinamentos aos professores, esses treinamentos se apresentam distantes das práticas pedagógicas dos profissionais e de suas condições de trabalho. Zanato et al. (2020), reforçam que a utilização das tecnologias é mais um instrumento de suporte em sala de aula, pois pode facilitar a visualização e apresentação do conteúdo ministrado favorecendo o ensino-aprendizagem, entretanto, tais autores alegam que, os professores devem ser estimulados com a formação continuada, para que os mesmos pensem pedagogicamente na utilização das TIC em sala de aula.

Kenski (2012) defende que os cursos de formação de professores devem ser os locais por excelência em que os alunos possam dispor de laboratórios de tecnologias educacionais onde se familiarizem com os recursos de comunicação. É preciso também se pensar em novos espaços - materialmente concretos ou virtuais - em que professores e alunos possam interagir com mais frequência com tecnologias. Cursos de extensão, videoconferências, softwares educativos e projetos multimídia de educação a distância, ajudariam a otimizar o uso dos recursos disponíveis. 
A formação continuada do professor é uma exigência, pois não termina, principalmente neste contexto em que as tecnologias se transformam rapidamente. Há necessidade de as escolas repensarem suas propostas, inclusive dos espaços formativos que precisam priorizar momentos de atualização e de reconstrução de concepções, de forma que os educadores possam não apenas "dominar" o uso de um recurso tecnológico, mas possa transpor didaticamente, com a utilização de mídias e tecnologias diversas, os conteúdos curriculares ou não nas rotinas pedagógicas (Alarcão, 2001).

Assim, deve-se avaliar com cuidado e acompanhar de perto a implantação ou adoção das TIC no ambiente educacional, de forma que elas venham possibilitar o crescimento e o desenvolvimento do potencial humano e científico dos alunos, mas que não sejam vistas ou tomadas como um fim em si mesmas.

As mudanças que chegam à escola, não correspondem apenas a um aperfeiçoamento do sistema educacional. Ela exige uma transformação profunda, que imponha, obrigatoriamente, a implantação de políticas educacionais coerentes com as transformações da sociedade como um todo, e não, simplesmente, modernizadoras. Desta forma, as TIC não devem ser vistas sob a perspectiva de simples instrumentos metodológicos modernos os quais podem ser implantados de forma isolada e desarticulada, mantendo crianças, jovens, adolescentes e professores como meros consumidores de um conhecimento pronto que passa agora a circular e ser entregue via as ditas novas tecnologias (Pretto, 2003).

Há que se destacar que a ação docente mediada pelas tecnologias é uma ação partilhada. Já não depende apenas de um único professor, isolado em sua aula, mas das interações que forem possíveis para o desenvolvimento das situações de ensino. Alunos, professores e tecnologias interagindo com o mesmo objetivo geram um movimento revolucionário de descobertas e aprendizados. Essa formulação já mostra que a instrumentação técnica é uma parte muito pequena do aprendizado docente para a ação bem-sucedida na mediação entre educação e tecnologias.

Além disso, é essencial que tenhamos consciência de que sua integração à educação já não é uma opção: estas tecnologias já estão no mundo, transformando algumas dimensões da vida social e econômica, cabe ao campo educacional integrá-las e tirar de suas potencialidades comunicacionais e pedagógicas o melhor proveito.

É preciso levar em conta que as tecnologias não são outra senão recursos cuja função e auxiliar o trabalho humano, facilitá-lo e maximizar seus efeitos. A base disso é o modo de ser do trabalho humano que consiste numa atividade adequada a finalidades (Saviani, 2011).

\section{Considerações Finais}

Reconhecer as diferenças individuais dos alunos é um conceito básico quando os professores se preparam para ensinar. É uma suposição fundamental de ensino estratégico e aprendizagem, pois, ao que escolhemos ensinar na sala de aula deve ser uma interação do que sabemos sobre as variáveis de instrução, aprendizagem, desempenho e fatores contextuais. Neste contexto, o processo educativo deve ser orientado por metodologias que permitam atender aos objetivos propostos pelos docentes, e tais metodologias devem ser utilizadas com o propósito de alcançar com eficácia os objetivos de ensinoaprendizagem.

De acordo com Dyer \& Osborne (1996), os alunos vêm de diferentes origens e têm experiências e habilidades variadas, sobretudo no que se refere ao uso de tecnologias. O bom ensino não depende apenas de estratégias de ensino ou de sua eficácia, mas também depende das necessidades individuais e da adequação do conteúdo. A seleção de uma apropriada abordagem de ensino é um dos processos mais importantes para ter sucesso no ensino e desempenho dos alunos.

É imprescindível discutir as abordagens necessárias para capacitar o processo de indução e inovação constantes na educação e aprendizagem. Apesar do uso da tecnologia estar sujeito à forma como os materiais auxiliam o educador no enriquecimento do procedimento de instrução, seu sucesso está ligado à confiança do educador no valor da sua utilização, caso a tecnologia esteja razoavelmente disponível. 
Alcançar o objetivo de integração significativa da tecnologia na sala de aula, ou seja, usar a tecnologia para apoiar o ensino e a aprendizagem do século XXI, não depende apenas de fatores relacionados à tecnologia, as crenças pedagógicas pessoais dos professores desempenham um papel fundamental em suas decisões pedagógicas sobre como integrar a tecnologia em suas práticas de sala de aula.

O grande desafio está em encontrar formas positivas e viáveis de integrar as TIC no processo de ensino-aprendizagem, no quadro dos currículos atuais, da situação profissional dos professores e das condições concretas de atuação em cada escola.

\section{Referências}

Acosta, M. A. F., Rodríguez, J. M. \& González, D. O. (2021). Use of Bioinformatics Technologies and Databases to Teach Analysis of Genetic Sequences to Undergraduate Students in Physics, Biotechnology, and Biology: The Specific Case of the SARS-CoV-2 Spike Protein. Creative Education, $12,193-202$.

Ajjan, H. \& Hartshorne, R. (2008). Investigating faculty decisions to adopt Web 2.0 technologies: Theory and empirical tests. The Internet and Higher Education, 11(2), 71-80.

Akram, S. \& Malik, K. (2012). Use of audio-visual aids for effective teaching of biology secondary school level. Journal Elixir Leadership Mgmt, 34(2), 10597-10605

Alarcão, I. (2001). Escola Reflexiva e a Nova Racionalidade. Artmed Editora.

Anderson, J., Van Weert, T. \& Duchâteau, C. (2002). Information and communication technology in education: A curriculum for schools and programme of teacher development (UNESCO).

Arroyo, C. G. (2011). On-line social networks: innovative ways towards the boost of collaborative language learning. Ict for language learning.

Aydin, S. \& Yel, M. (2011). The effect of brain-based learning biology education upon the academic success and attitude. Energy Education Science and Technology Part B, 3(1), 87-98.

Bamiah, S. N., Brohi, S. N. \& Rad, B. B. (2018). Big data technology in education: Advantages, implementations, and challenges. Journal of Engineering Science and Technology, 13, 229-241.

Barrio, C. M., Muñoz-Organero, M. \& Soriano, J. S. (2015). Can gamification improve the benefits of student response systems in learning? An experimental study. IEEE Transactions on Emerging Topics in Computing, 4(3), 429-438.

Belloni, M. L. (2002). Ensaio sobre educação à distância no Brasil. Educação \& Sociedade, 23(78), 117-142.

Berrett, B., Murphy, J. \& Sullivan, J. (2012). Administrator insights and reflections: Technology integration in schools. Qualitative Report, 17(1), 200-221.

Bingimlas, K. (2009). Barriers to the successful integration of ICT in teaching and learning environments: A review of the literature. Eurasia Journal of Mathematics. Science \& Technology Education, 5(3), 235-245.

Bolton, R., Parasuraman, A., Hoefnagels, A., Migchels, N., Kabadayi, S., Gruber, T., Komarova, Y. \& David, S. (2013). Understanding generation Y and their use of social media: A review and research agenda. Journal of Service Management, 24(3), 245-267.

Bozkaya, M., Erdem-Aydin, İ. \& Genc-Kumtepe, E. (2012). Research trends and issues in educational technology: A content analysis of TOJET (2008-2011). The Turkish Online Journal of Educational Technology, 11(2), 264-277.

Cheong, C., Cheong, F. \& Filippou, J. (2013). Quick Quiz: A Gamified Approach for Enhancing Learning. In Pacis (p. 206).

Costley, K. C. (2014). The Positive Effects of Technology on Teaching and Student Learning. Online submission. https://eric.ed.gov/?id=ED554557

Cunha, M. D. \& Bizelli, J. L. (2015). Inovações tecnológicas e contexto escolar: reflexões necessárias. Revista Ibero-Americana de Estudos em Educação, $10(1), 50-66$.

Dyer, J. E. \& Observe, E. W. (1996). Effects of teaching approach on achievement of agricultural education students with varying learning styles. Journal of Agricultural Education, 37, 43-51.

Ertmer, P. A., Ottenbreit-Leftwich, A. T. \& Tondeur, J. (2015). Teachers' beliefs and uses of technology to support 21 st-century teaching and learning. International handbook of research on teacher beliefs, 403.

Fives, H. \& Gill, M. G. (Eds.). (2014). International handbook of research on teachers' beliefs. Routledge.

Graham, K. (2015). Tech Matters: Getting into Kahoot(s): Exploring a game-based learning system to enhance student learning. Loex Quarterly, $42(3), 4$.

Gross, D., Pietri, E. S., Anderson, G., Camihort, K. M., Graham, M. J. (2015). Increased preclass preparation underlies student outcome improvement in the flipped classroom. CBE-Life Sciences Education, 14(4), 36.

Hannafin, M. J. \& Hill, J. R. (2002). Epistemology and the design of learning environments. Trends and issues in instructional design and technology, p.70-82.

Huang, R., Spector, J. M. \& Yang, J. (2019). Educational Technology a Primer for the 21st Century. Springer. 
Inan, F. A. \& Lowther, D. L. (2010). Factors affecting technology integration in K-12 classrooms: A path model. Educational technology research and development, 58(2), 137-154.

Incantalupo, L., Treagust, D. F. \& Koul, R. (2014). Measuring student attitude and knowledge in technology-rich biology classrooms. Journal of Science Education and Technology, 23(1), 98-107.

Kelley, P. \& Whatson, T. (2013). Making long-term memories in minutes: a spaced learning pattern from memory research in education. Frontiers in human neuroscience, $7,589$.

Kenski, V. M. (2012). Educação e Tecnologias: o novo ritmo da informação. (8a ed.), Papirus Editora.

Lawless, K. A. \& Pellegrino, J. W. (2007). Professional development in integrating technology into teaching and learning: Knowns, unknowns, and ways to pursue better questions and answers. Review of educational research, 77(4), 575-614.

Mammes, I. (2004). Promoting girls' interest in technology through technology education: A research study. International Journal of technology and design Education, 14(2), 89-100.

Mayes, R. (2011). Themes and Strategies for Transformative Online Instruction: A Review of Literature. In: Global Learn. Association for the Advancement of Computing in Education (AACE). p. 2121-2130.

Mazman, S. G., Usluel, Y. K. \& Çevik, V. (2009). Social influence in the adoption process and usage of innovation: Gender differences. International Journal of Behavioral, Cognitive, Educational and Psychological Sciences, 1(4), 229-232.

McLuhan, M. (1996). Os meios de comunicação como extensões do homem. Editora Cultrix.

Nicolle, P. S. \& Lou, Y. (2008). Technology adoption into teaching and learning by mainstream university faculty: A mixed methodology study revealing the "how, when, why, and why not". Journal of Educational Computing Research, 39(3), 235-265.

Ochoa, C. J. (2016). Are we ready for a disruptive innovation in education? Australasian Journal of Educational Technology, 22, 39-63.

Olaore, I. B. (2014). The impacts (positive and negative) of ICT on education in Nigeria. Developing Country Studies, 4(23), 154-156.

Oliveira, A., Behnagh, R. F., Ni, L., Mohsinah, A., Burgess, K. J. \& Guo, L. (2019). Emerging technologies as pedagogical tools for teaching and learning science: A literature review. Human Behavior and Emerging Technologies, 1(2), 149-160.

Pereira, A. S., Shitsuka, D. M., Parreira, F. J. \& Shitsuka, R. (2018). Metodologia da pesquisa científica. UFSM.

Pretto, N. (2003). Educação e inovação tecnológica: Um olhar sobre as políticas públicas brasileiras. Revista Pedagógica, 5(11), 65-84.

Roehl, A., Reddy, S. L. \& Shannon, G. J. (2013). The flipped classroom: An opportunity to engage millennial students through active learning strategies. Journal of Family \& Consumer Sciences, 105(2), 44-49.

Ross, S. M., Morrison, G. R. \& Lowther, D. L. (2010). Educational technology research past and present: Balancing rigor and relevance to impact school learning. Contemporary Educational Technology, 1(1), 17-35.

Santos, J. R. \& Souza, B. T. C. (2019). A Utilização das Tecnologias da Informação e Comunicação no Ensino de Biologia: uma Revisão Bibliográfica. ID on line Revista de Psicologia, 13(45), 40-59.

Saviani, D. (2011). Educação em diálogo. Autores Associados - coleção Memória da educação.

Sivin, J. P., Bialo, E. \& Langford, J. (2000). 2000 research report on the effectiveness of technology in schools. SIIA.

Spector, J. M. (Ed.). (2015). The SAGE encyclopedia of educational technology. Sage Publications.

Thompson, K. V., Nelson, K. C., Marbach-Ad, G., Keller, M. \& Fagan, W. F. (2010.). Online interactive teaching modules enhance quantitative proficiency of introductory biology students. CBE-Life Sciences Education, 9 (3), 277-283.

Tondeur, J., Valcke, M. \& Van Braak, J. (2008). A multidimensional approach to determinants of computer use in primary education: Teacher and school characteristics. Journal of Computer Assisted Learning, 24 (6), 494-506.

Waldrop, L. D., Adolph, S. C., Diniz Behn, C. G., Braley, E., Drew, J. A., Full, R. J., Gross, L. J. \& Shtylla, B. (2015). Using active learning to teach concepts and methods in quantitative biology. Interactive and Comparative Biology, 55(5), 933-948.

Wang, A. I. \& Lieberoth, A. (2016). The effect of points and audio on concentration, engagement, enjoyment, learning, motivation, and classroom dynamics using Kahoot. In: European Conference on Games Based Learning. Academic Conferences International Limited.

Ward, R., Moule, P. \& Lockyer, L. (2006). Adoption of Web 2.0 Technologies in Education for Health Professionals in the UK: Where Are We and Why? Electronic Journal of e-Learning, 7(2), 165-172.

Yalim, E. (2021). High school students' level of knowledge and attitudes towards the use of technology in biology classrooms. Tese de Doutorado. Bilkent University.

Yassanne, G. L. (2014). Integrating computer technology in the teaching of Biology. International Journal Of Biology Education, 3, 2.

Zanato, A. R., Strieder, D. M. \& Campos, T. A. (2020) Challenges for the use of ICTs in the classroom: perception of Nature Science teachers. Research, Society and Development, 9(11), 1-24. 